\title{
Sammelerklärung
}

\section{Vorsicht bei der Unterschrift}

\author{
Unterschreibt ein dazu nicht berechtigter Arzt die Sammelerklärung, \\ kann das zum Verlust des gesamten Honorars führen. Insbesondere \\ medizinische Versorgungszentren laufen aufgrund ihrer Organisations- \\ struktur Gefahr, diesen Fehler zu begehen.
}

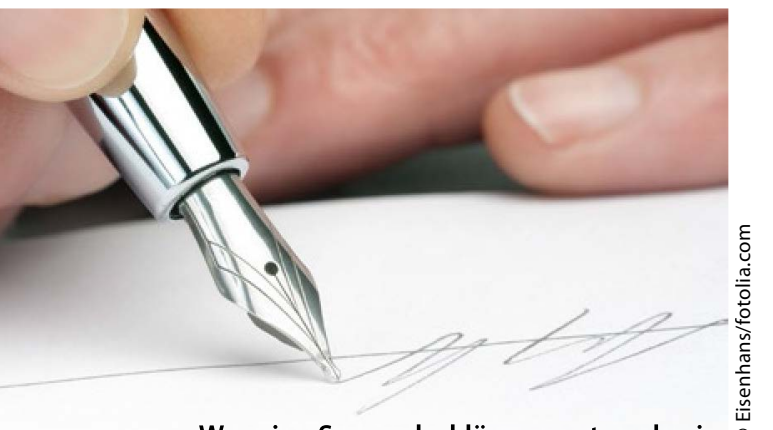

Wer eine Sammelerklärung unterschreiben darf, steht meistens auf dem Formular selbst. Es lohnt aber auch der Blick in die Abrechnungsordnung der Kassenärztlichen Vereinigung und in den Honorarverteilungsmaßstab.

Die mit der Quartalsabrechnung obligatorisch einzureichende Bestätigung der bestimmungsgemäßen und genehmigungskonformen Abrechnung wird als Sammelerklärung bezeichnet. Wer die darauf erforderliche Unterschrift leisten muss, regelt der Honorarverteilungsmaßstab (HVM) oder eine gesonderte Abrechnungsordnung der jeweiligen kassenärztlichen Vereinigung (KV).

In der Regel ist auch auf der Sammelerklärung selbst aufgeführt, wer unterschriftsberechtigt ist. Die Missachtung dieser Vorgabe ist nun einem medizinischen Versorgungszentrum (MVZ) zum Verhängnis geworden. Nach den Vorgaben des HVM musste der ärztliche Leiter unterschreiben, tatsächlich hatte jedoch der Geschäftsführer der MVZ$\mathrm{GmbH}$ unterzeichnet.

\section{GmbH-Argument überzeugt nicht}

Das Landessozialgericht NordrheinWestfalen hat nun in einem einstweili- gen Rechtsschutzverfahren festgestellt, dass die Unterschrift des Geschäftsführers gegen den $H V M$ verstieß und damit rechtswidrig war. Die KV war laut $\mathrm{Ge}$ richt zur Aufhebung der Honorarbescheide berechtigt und darf das Honorar vollständig zurückfordern (LSG Essen, Az.: L11 KA 58/15 ER).

Im Zusammenhang mit der Abrechnung ärztlicher Leistungen spricht das Bundessozialgericht bereits seit Jahren von der Pflicht zur "peinlich genauen Abrechnung“, denn der Teufel steckt wie immer im Detail. Wie die jetzige Entscheidung des LSG Nordrhein-Westfalen bestätigt, ist dies wörtlich zu nehmen. Nur diejenige Person, die nach den Abrechnungsvorschriften die Sammelerklärung unterschreiben muss, darf diese auch unterschreiben. Wenn dies der ärztliche Leiter ist, scheidet eine Unterschrift durch den Geschäftsführer oder andere Personen aus.

Das betroffene MVZ hatte in dem gerichtlichen Verfahren damit argumentiert, dass eine $\mathrm{GmbH}$ gerichtlich und außergerichtlich durch ihren $\mathrm{Ge}$ schäftsführer vertreten werde und dieser allein dazu berechtigt sei, Willenserklärungen für die $\mathrm{GmbH}$ abzugeben. Aus diesem Grund müsse der Geschäftsführer auch die Sammelerklärung unterschreiben, weshalb die abweichende Regelung im Honorarverteilungsmaßstab rechtswidrig sei.

Das Landessozialgericht ist dieser Argumentation nicht gefolgt. Es legt den Schwerpunkt darauf, dass die Sammelerklärung eine Wissenserklärung mit einer Verantwortungszuweisung ist. Wissenserklärungen sind rechtlich keine Willenserklärungen.
Mit der Unterschrift unter die Sammelerklärung übernehme der ärztliche Leiter Verantwortung für die Richtigkeit. Eine solche Zuweisung im HVM sei insbesondere deshalb zulässig, weil jedes MVZ einen ärztlichen Leiter haben müsse. Ob daneben eine andere leitende Person oder ein Geschäftsführer bestehe, sei sozialrechtlich nicht vorgeschrieben. Gesellschaftsrechtlich könnte ein MVZ in der Rechtsform einer $\mathrm{GmbH}$ sogar zeitweilig führungslos, also ohne Geschäftsführer, sein.

\section{Bei Vertretung absichern}

Auch wenn die Entscheidung in einem Verfahren des vorläufigen Rechtsschutzes erging, spricht doch alles dafür, dass sie Bestand haben wird. Es kann auch nicht ausgeschlossen werden, dass die KVen künftig sehr genau prüfen werden, wer die Sammelerklärung unterschrieben hat. Jedes MVZ sollte also „peinlich genau" darauf achten, dass tatsächlich der ärztliche Leiter unterschreibt, wenn dessen Unterschrift auf der Sammelerklärung für den jeweiligen KV-Bezirk vorgeschrieben ist.

Die KVen legen in der Regel auch fest, dass bei einer Abwesenheit des ärztlichen Leiters aus den in $\$ 32$ der Zulassungsverordnung für Ärzte (Ärzte-ZV) genannten Gründen ein Vertreter unterschreiben darf. Sollte dies allerdings im HVM, der Abrechnungsordnung oder im Text der Sammelerklärung nicht ausdrücklich erwähnt sein, so sollte im Vertretungsfall zuvor unbedingt eine Rücksprache und Abstimmung mit der KV erfolgen.

Die Vereinbarung über Vordrucke für die vertragsärztliche Versorgung enthält aktuell 45 verschiedene Formulare, die ein Arzt unterschreiben muss. Viermal im Jahr ist aber eben ein Formular besonders wichtig: Das gesamte Quartalshonorar kann von der richtigen Unterschrift unter die Sammelerklärung abhängen.

Dr. Ingo Pflugmacher 